\title{
A Dynamic Tap Allocation for Concurrent CMA-DD Equalizers
}

\author{
Diego von B. M. Trindade, Vitor Halmenschlager, Leonardo Ortolan, Maria C. F. De Castro, \\ Fernando C. C. De Castro, and Fabrício Ourique
}

Centro de Pesquisa em Tecnologias Wireless (CPTW), Pontifícia Universidade Católica do Rio Grande do Sul (PUCRS), Avenda Ipiranga 6681, 90619-000 Porto Alegre, RS, Brazil

Correspondence should be addressed to Fabrício Ourique, fourique@gmail.com

Received 10 August 2010; Revised 23 September 2010; Accepted 20 October 2010

Academic Editor: Christoph F. Mecklenbräuker

Copyright (C) 2010 Diego von B. M. Trindade et al. This is an open access article distributed under the Creative Commons Attribution License, which permits unrestricted use, distribution, and reproduction in any medium, provided the original work is properly cited.

This paper proposes a dynamic tap allocation for the concurrent CMA-DD equalizer as a low complexity solution for the blind channel deconvolution problem. The number of taps is a crucial factor which affects the performance and the complexity of most adaptive equalizers. Generally an equalizer requires a large number of taps in order to cope with long delays in the channel multipath profile. Simulations show that the proposed new blind equalizer is able to solve the blind channel deconvolution problem with a specified and reduced number of active taps. As a result, it minimizes the output excess mean square error due to inactive taps during and after the equalizer convergence and the hardware complexity as well.

\section{Introduction}

The Concurrent Equalizer (CEQ) is based on a concurrent architecture which comprises the classical direct decision (DD) equalizer and Godard's widespread known constant modulus (CMA) blind equalizer [1]. In the CEQ architecture, the DD branch is coordinated by the CMA branch gradient trajectory. Since the CEQ proposition in 2001 [2], several contributions have been reported [3-5], to name a few. A significant complexity reduction is achieved when both the DD-updated filter and the CMA-updated filter are replaced by one single equivalent FIR filter located after the sum block in the original concurrent split architecture, as shown in Figure 1. Notice that the minimization of the Euclidean distance-based $J_{\mathrm{DD}}$ cost function is controlled by a nonlinear directional link between $J_{\mathrm{CMA}}$ and $J_{\mathrm{DD}}$, where $J_{\mathrm{CMA}}=(1 / 4) E\left\{\left(|y|^{2}-\gamma\right)^{2}\right\}, J_{\mathrm{DD}}=(1 / 2) E\left\{|Q\{y\}-y|^{2}\right\}, y$ is the equalizer output, $\gamma$ is the CMA dispersion constant, $Q\{\cdot\}$ is the operator which returns the reference constellation IQ symbol with the smallest Euclidean distance to the argument, and $E\{\cdot\}$ is the statistical expectancy operator [6]. The nonlinear directional link controls $J_{\mathrm{DD}}$ minimization such that it only takes place when the minimization of the energy dispersion-based $J_{\mathrm{CMA}}$ cost function is judged to have achieved a successful adjustment with high certainty.
Certainty is measured as the closeness of the output to the same IQ symbol in the reference constellation before and after a perturbation is imposed to the equalizer [2].

Let $\mathbf{B}=\left[\begin{array}{llll}B_{0} & B_{1} & \cdots & B_{L-1}\end{array}\right]^{T}$ be the vector whose components $B_{k}$ represent the taps of the CMA\&DD-updated FIR filter shown in Figure 1 and let $\mathbf{r}=\left[\begin{array}{llll}r_{0} & r_{1} & \cdots & r_{L-1}\end{array}\right]^{T}$ be the vector which defines the channel regressor, where $L$ is the equalizer length [2]. The components $r_{k}$ of the $n$th regressor $\mathbf{r}(n)$ are $T / 2$-spaced noisy samples received from the channel, where an even $k$ index refers to an on-baud sample. $T$ is the baud interval and $k=0,1, \ldots, L-1$. Thus, the governing algorithm for the fractionally spaced [2] CEQ of Figure 1 is as shown in Algorithm 1.

Algorithm 1. CEQ algorithm with one single FIR filter. $\gamma=$ $E\left\{|A|^{4}\right\} / E\left\{|A|^{2}\right\}$ is the CMA dispersion constant [6]. $A$ is the IQ symbol alphabet. $Q\{\cdot\}$ returns the IQ symbol from $A$ with the smallest Euclidean distance to the argument. $\eta_{\mathrm{CMA}}$ and $\eta_{\mathrm{DD}}$ are the gradient step sizes.

\section{CEQ Algorithm}

Step 1. $n=0$ \& init $\mathbf{B}(0)$

Step 2. $y(n)=\mathbf{B}^{T}(n) \mathbf{r}(n)$ 


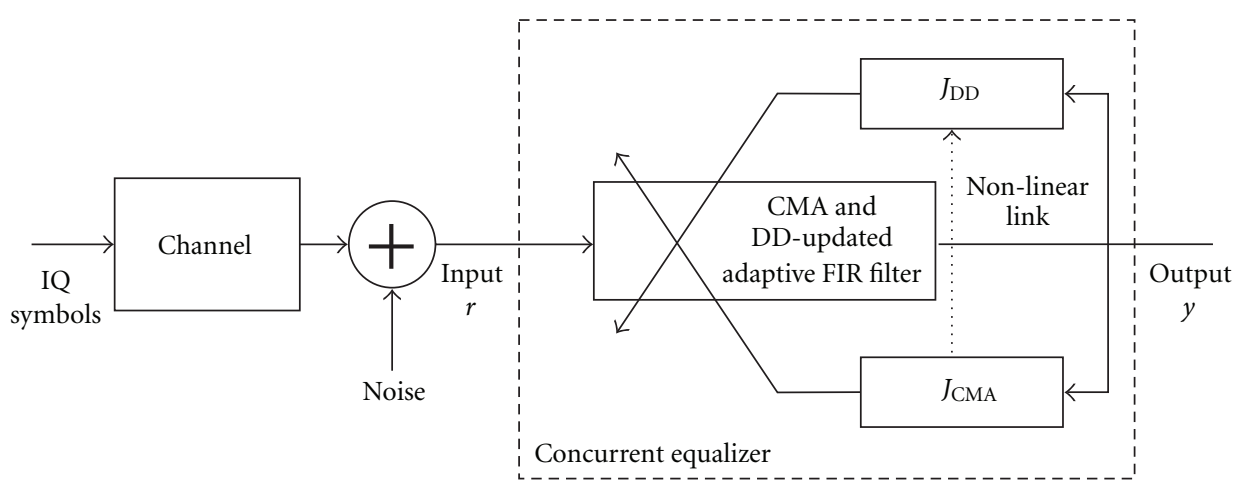

Figure 1: CEQ equivalent baseband model.

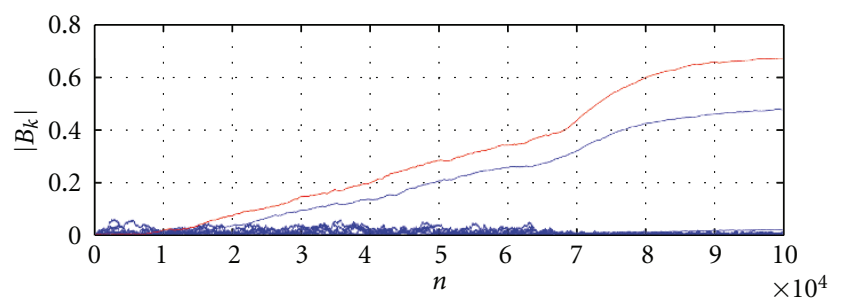

(a)

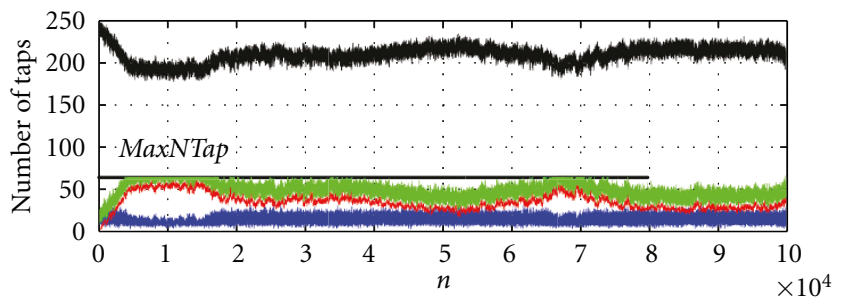

(b)

FIgure 2: Curves for "Brazil A" profile Table 1 with $150 \mathrm{~Hz}$ Doppler rotation, SNR $=30 \mathrm{~dB} . \sigma=\left(D_{\min } / \gamma\right)^{2}=0.015$ is the MSE convergence level, $D_{\min }=\left|s_{k}-s_{k-1}\right| / 2, s_{k} \in A . L=256$, FIR init $@ B_{L / 2}=1.0, \eta_{\mathrm{CMA}}=3 \times 10^{-4}$, and $\eta_{\mathrm{DD}}=10 \eta_{\mathrm{CMA}}$. MaxNTap $=64, \alpha_{\max }=16$, and $\xi=3 \times 10^{-3}$. (a) CEQ filter tap magnitude value $\left|B_{k}\right|$ in the range $k=0,1, \ldots, 9, L=256$. (b) CEQTR tap rank distribution.

TABLE 1: "Brazil A" channel multipath profile.

\begin{tabular}{lcccccc}
\hline \multirow{2}{*}{ Description } & \multicolumn{7}{c}{ Path } \\
& 1 & 2 & 3 & 4 & 5 & 6 \\
\hline Delay $(\mu s)$ & 0.00 & 0.15 & 2.22 & 3.05 & 5.86 & 5.93 \\
Gain $(\mathrm{dB})$ & 0.0 & -13.8 & -16.2 & -14.9 & -13.6 & -16.4 \\
\hline
\end{tabular}

Step 3. $\mathbf{B}(n+1)=\mathbf{B}(n)+\eta_{\text {CMA }} y(n)\left(\gamma-|y(n)|^{2}\right) \mathbf{r}^{*}(n)$

Step 4. $\tilde{y}(n)=\mathbf{B}^{T}(n+1) \mathbf{r}(n)$

Step 5. $\mathbf{B}(n+1)=\mathbf{B}(n+1)+\eta_{\mathrm{DD}}[Q\{y(n)\}-y(n)] \mathbf{r}^{*}(n)$ if $Q\{y(n)\}=Q\{\tilde{y}(n)\}$

Step 6. $n=n+1$

Step 7. GOTO Step 2.

For digital television (DTV) implementation, the sparse nature of the broadcast channel suggests the use of a dynamic tap allocation (DTA) algorithm, not only as a means to reduce the equalizer complexity, but also as a means to minimize the excess output mean squared error (MSE). Several algorithms have been proposed to this end [7-11]. A detailed survey is presented by Wei et al. [12]. Among the low complexity methods, Fan et al. [13] proposed that the dynamics of the allocation process should be determined by the taps magnitude.
In this paper, we propose a DTA suited for the CEQ and based on a ranking procedure which ranks the filter taps according to three fitness levels $\{-1,0,1\}$ determined from the tap magnitudes compared to a fixed threshold, thus avoiding the complexity of magnitude ordering, adopted in some proposals.

\section{Tap Ranking and Dynamic Allocation}

As in any gradient-based algorithm, the CEQ gradient trajectory wanders around the minimum of the $J_{\mathrm{CMA}}$ and $J_{\mathrm{DD}}$ functions as a consequence of the adaption noise [12], increasing the output MSE during and after the convergence. Given a channel profile, the adaption noise is generated by those filter taps whose values present a random behavior along time. Such randomness stems from the fact that these taps are uncorrelated with the $J_{\mathrm{CMA}}$ and $J_{\mathrm{DD}}$ gradient minimization for the given channel. On the other hand, taps which are correlated with the gradient dynamics present a nearly monotonic value behavior along time.

For example, Figure 2(a) shows the behavior of FIR filter taps $B_{0}-B_{9}$ when the CEQ is operating under the "Brazil A" DTV channel profile [14] shown in Table 1. We assume an 8-VSB ATSC [15] baseband sequence uniformly drawn from the unit variance alphabet $A=$ $\{1.528,1.091,0.655,0.218,-0.218,-0.655,-1.091$, $1.528\}$ with $\gamma=1.762$ [15]. The baud rate is $f_{s}=10.762 \mathrm{MHz}$ and the baud interval is $T=1 / f_{s}$. The signal-to-noise ratio (SNR) at the equalizer input is set to $30 \mathrm{~dB}$. 
Except for the active taps $B_{0}$ and $B_{2}$, which increase monotonically until steady state is reached, all other taps in the range are inactive, since they present a random magnitude value behavior. Inactive taps play no effective and sustained role in the $J_{\mathrm{CMA}}$ and $J_{\mathrm{DD}}$ gradient minimization procedure. Intrinsic to the CEQ operation is the larger gradient step size $\left(\eta_{\mathrm{DD}} \approx 10 \eta_{\mathrm{CMA}}\right)$ for the $\mathrm{DD}$ branch. Therefore, since the larger $\mathbf{B}$ update generated by the DD branch is certainty-activated along time, it imposes a strong trend on the $\mathbf{B}$ components (taps) $B_{k}$ which reinforces the distinction between monotonic and random tap behavior along the gradient trajectory. Thus a fixed magnitude threshold $\xi$ separates the taps in two well-defined classesactive and inactive.

To determine which of the equalizer taps are active or inactive, the $L$ taps are ranked in three levels of hierarchy $\{-1,0,1\}$, along the lines of genetic algorithms. Active tapsthose subject to the gradient update and that contribute to the output $y$-are taps which belong to rank 1 and rank 0 hierarchies. Inactive taps belong to rank -1 hierarchy, and therefore are deactivated in all steps on Algorithm 1. The most fitted taps are that ones with magnitude greater than threshold $\xi$, and thus belong to rank 1 hierarchy. Rank 0 taps-independently of their magnitudes-are randomly picked with a low probability $0.05<p_{0}<0.10$. The parameter $p_{0}$ plays a similar role here as the mutation factor does in genetic algorithms. That is, a small number of $L$ taps can be considered as active, given that the total number of rank 1 and rank 0 taps is less than an arbitrary Max NTap $<L$. The random picking of taps is necessary when operating under a dynamic multipath scenario, that is, when the receiver is under mobile operation. A quantitative measure of the multipath dynamics is the Doppler deviation. Under mobile operation, the channel impulse response varies periodically with a period given by approximately the inverse of the Doppler frequency. Thus, the channel frequency domain transfer function varies accordingly. Since the equalizer should ideally implement the channel inverse transfer function in order to cancel the multipath effects, it follows that the equalizer taps must track the channel variations at nearly the Doppler rate. The DTA procedure reinforces the largest magnitude taps during the gradient convergence phase, and this action interlocks the active tap set even after the equalizer convergence. Therefore, when the channel is time variant, as is the case under mobile operation, it is necessary to refresh the active tap set population via random picking in order to cope with the dynamic channel. Algorithm 2 shows the proposed DTA.

Algorithm 2 (DTA procedure).

\section{Tap Ranking and Dynamic Allocation}

Step 1. The rank $\chi_{k} \in\{-1,0,1\}$ of each tap $B_{k}, k=$ $0,1, \ldots, L-1$, is obtained according to

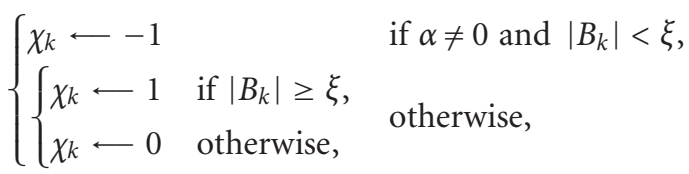

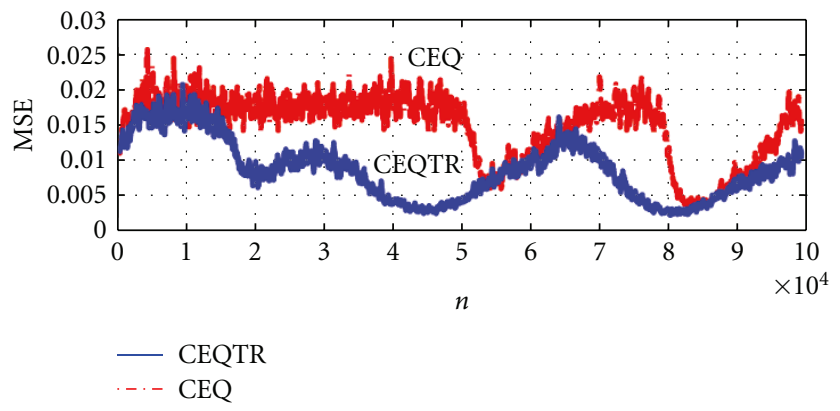

(a)

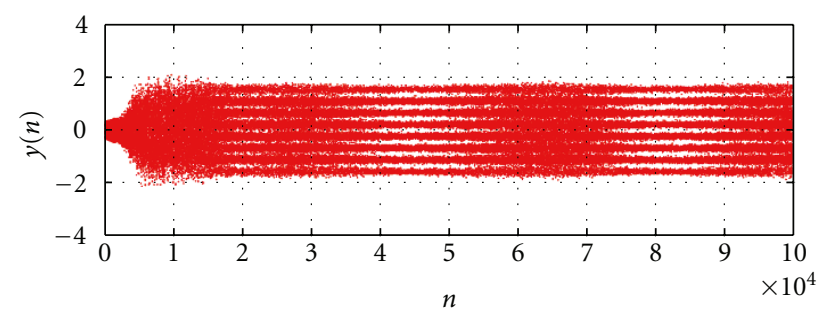

(b)

Figure 3: Curves with simulation parameters as in Figure 2. (a) CEQ and CEQTR output MSE. (b) CEQTR output $y$ corresponding to (a) MSE curve.

where $\alpha$ is a random integer draw with probability $p_{0}$ from the set $\left\{0,1, \ldots, \alpha_{\max }-1\right\}$, with $p_{0}=1 / \alpha_{\max } . \xi$ is the magnitude threshold.

Step 2. Each tap $\mathbf{B}_{k}$ with rank $\chi_{k}=1$ is labeled as "active" up to a maximum number MaxNTap of active taps.

Step 3. Each tap $\mathbf{B}_{k}$ with rank $\chi_{k}=0$ is labeled as "active" up to a maximum number MaxNTap of active taps.

\section{Simulation Results}

In order to evaluate the proposed DTA method for operation under dynamic DTV channels, we vary the magnitude of the largest echo in the channel discrete impulse response according to $\cos \left(\pi\left(f_{\text {doppler }} / f_{s}\right) m\right), m$ is the $m$ th sample index in the $T / 2$-spaced baseband received sequence, and $f_{\text {doppler }}$ is the amount of the applied Doppler rotation. Denote as CEQTR the CEQ with filter taps ranked and allocated according to Algorithm 2 procedure. For the SER and MSE computation at least 50 runs are performed, and the average is taken.

Figures 3(a) and 3(b) show the operation with "Brazil A" profile for $f_{\text {doppler }}=150 \mathrm{~Hz}$ applied to $-13.6 \mathrm{~dB}$ echo. Notice that the CEQTR with a maximum 64 active taps not only does converge faster than the CEQ with 256 active taps but also attains a lower MSE under the same conditions. Notice in Figure 2(b) that the curve "active taps" is hard-limited to MaxNTap $=64$, thus reducing the complexity by a factor of L/MaxNTap. It also should be noted that MaxNTap is usually determined by hardware constraints, such as the number of DSP blocks available in the programmable logic device 


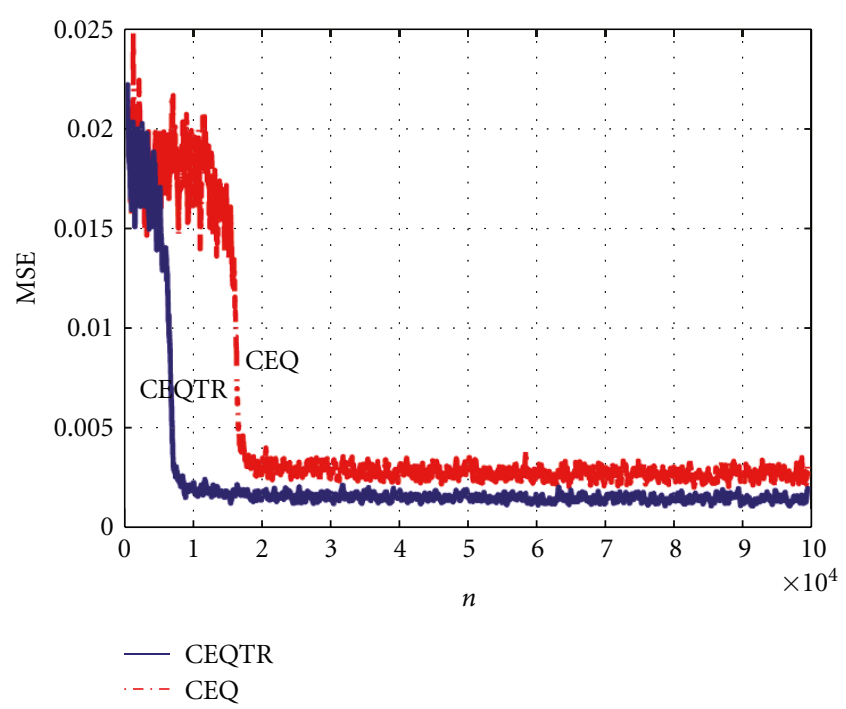

FIgUre 4: CEQ and CEQTR output MSE under "Brazil B" channel profile, no Doppler rotation applied, $\mathrm{SNR}=30 \mathrm{~dB} . L$, FIR init, $\eta_{\mathrm{CMA}}$, and $\eta_{\mathrm{DD}}$ as in Figure 2.

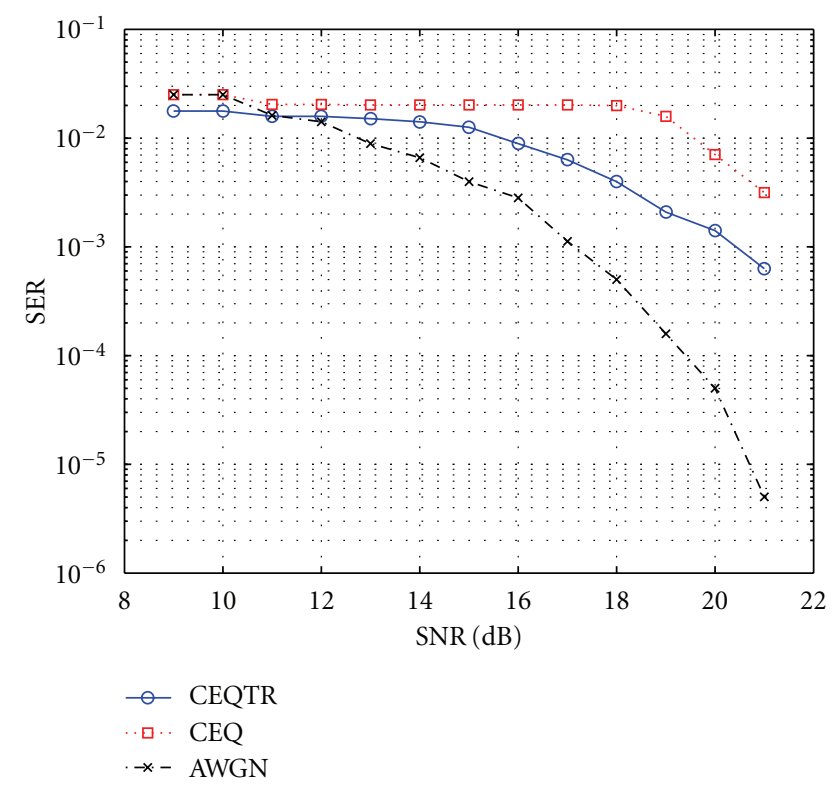

FIgURE 5: CEQ and CEQTR SER $\times$ SNR under "Brazil A" channel profile, no Doppler rotation applied. $L$, FIR init, $\eta_{\mathrm{CMA}}$, and $\eta_{\mathrm{DD}}$ as in Figure 2. AWGN refers to the CEQTR output SER for an AWGN [6] channel.

which runs the equalizer algorithm. In this paper, the DTA algorithm is executed at each received modulation symbol. However, it might be executed sparsely along time, at each received symbols. In this situation, we achieve a complexity reduction at the expense of a performance reduction, mainly under dynamic multipath operation.

For operation under static DTV channels, as is the case of the "Brazil B" profile in Table 2 [14], the CEQTR also outperforms the CEQ, as shown in Figure 4. It converges in

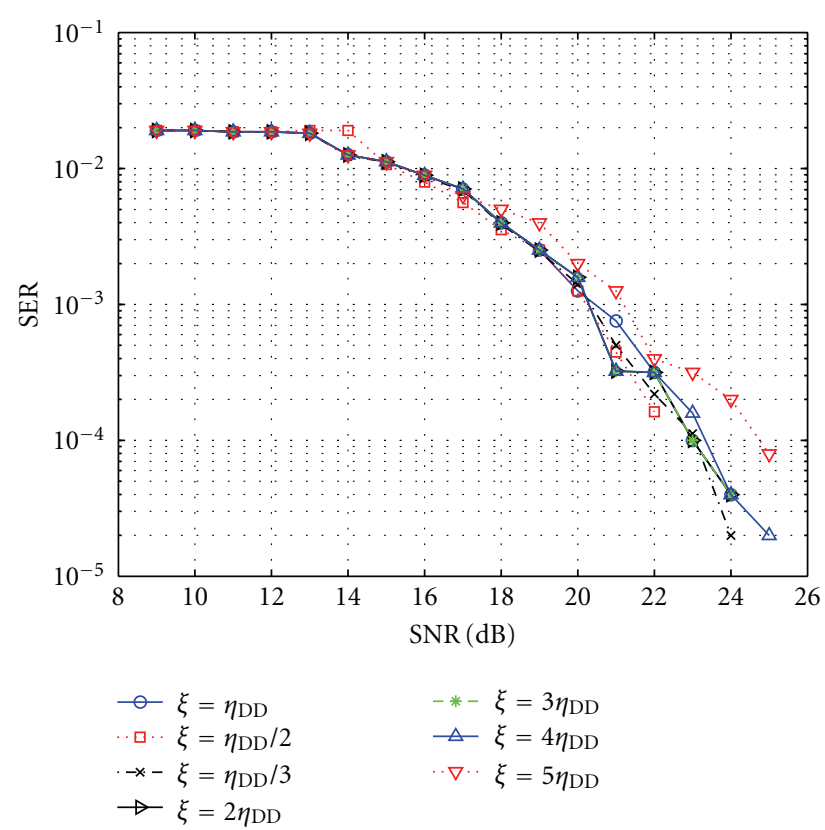

FIGURE 6: CEQTR SER $\times$ SNR having threshold $\xi$ as a parameter. "Brazil A" channel profile, no Doppler. Notice that the best performance is obtained for $\xi=\eta_{\mathrm{DD}}$, value also found for "Brazil B-E" profiles.

TABLE 2: "Brazil B" channel multipath profile.

\begin{tabular}{lcccccc}
\hline \multirow{2}{*}{ Description } & \multicolumn{7}{c}{ Path } \\
& 1 & 2 & 3 & 4 & 5 & 6 \\
\hline Delay $(\mu \mathrm{s})$ & 0.0 & 0.3 & 3.5 & 4.4 & 9.5 & 12.7 \\
Gain $(\mathrm{dB})$ & 0.0 & -12 & -4 & -7 & -15 & -22 \\
\hline
\end{tabular}

TABLE 3: ATSC R2.1 channel multipath profile.

\begin{tabular}{lcccccc}
\hline \multirow{2}{*}{ Description } & \multicolumn{7}{c}{ Path } \\
& 1 & 2 & 3 & 4 & 5 & 6 \\
\hline Delay $(\mu s)$ & 0.0 & -1.8 & 0.15 & 1.8 & 5.7 & 35 \\
Gain $(\mathrm{dB})$ & 0.0 & -14 & -14 & -4 & -8 & -12 \\
Phase or Doppler & $0^{\circ}$ & $125^{\circ}$ & $80^{\circ}$ & $45^{\circ}$ & $5 \mathrm{~Hz}$ & $90^{\circ}$ \\
\hline
\end{tabular}

less than half the time and achieves a nearly half MSE after convergence.

Simulations with "Brazil C", "D", and "E" DTV profiles [5] — not shown in this letter due to space limitationyielded similar results of Figure 4. It was also observed with these profiles that the CEQTR requires a much more "careless" initialization than the standard CEQ for a successful convergence, whether its filter is initialized or not in a position nearby the peak magnitude of the channel impulse response-position which is known to yield the fastest convergence.

Figure 5 shows the comparative symbol error rate (SER) under operation with "Brazil A" (Table 1) profile. It also shows the CEQTR SER for an AWGN [6] channel. Figure 6 shows the CEQTR SER sensitivity to the threshold $\xi$. 


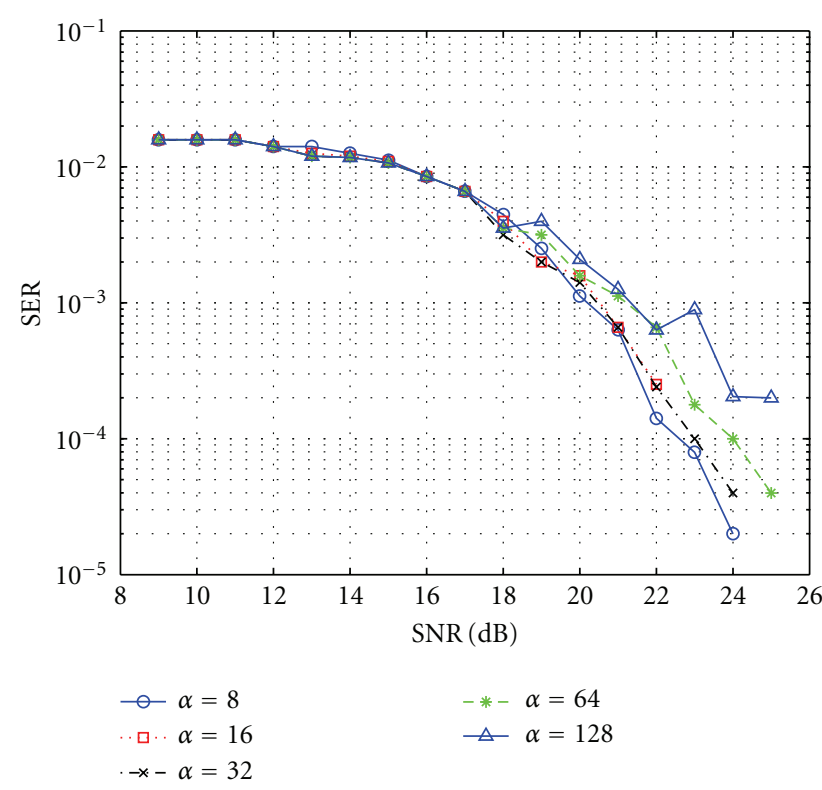

FIgure 7: CEQTR SER $\times$ SNR having $\alpha=\alpha_{\max }=1 / p_{0}$ as a parameter, with $p_{0}$ being the random tap picking probability in the DTA procedure of Algorithm 2. "Brazil A" channel profile, no Doppler. Notice that the best performance is obtained for $\alpha_{\max }=$ 16 , value also found for "Brazil B-E" profiles.

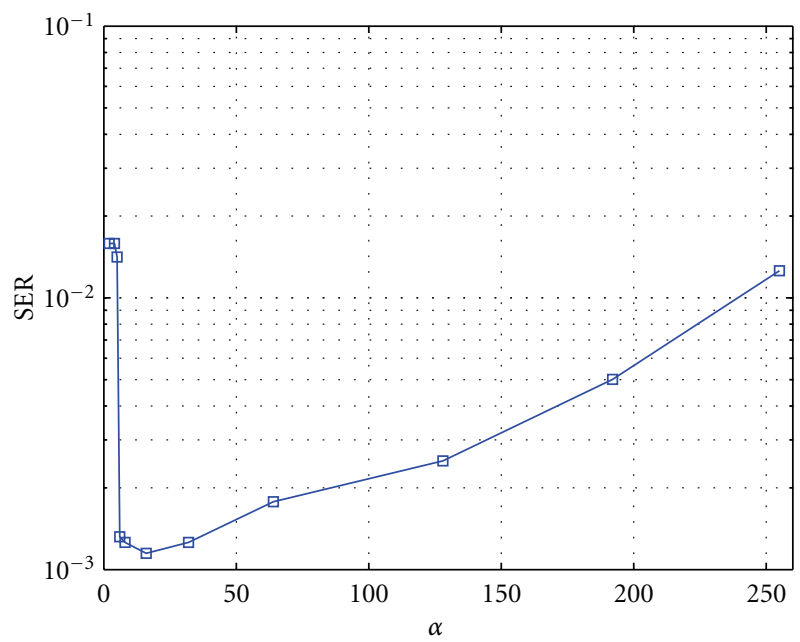

Figure 8: CEQTR SER $\times \alpha$, where $\alpha=\alpha_{\max }=1 / p_{0}$ (see Table 3). "Brazil A" profile with $150 \mathrm{~Hz}$ Doppler rotation and SNR $=20 \mathrm{~dB}$. Notice that the random tap picking probability $p_{0}=1 / \alpha_{\max }$ plays a significant role in the gradient convergence rate when Doppler effects are present in the channel.

Figures 7 and 8 show the SER sensitivity to the random tap picking probability $p_{0}=1 / \alpha_{\max }$ in the DTA procedure (see Algorithm 2); SER variation is almost independent of the value for $\alpha_{\max }$.

In Figure 9, we compare the proposed algorithm (CEQTR) with the algorithm presented in [13] (LS-DFE), under the ATSC R2.1 3\# channel (see Table 3). Notice that the CEQTR outperforms the LS-DFE for any SNR below $25 \mathrm{~dB}$. This behavior stems from the intrinsic error

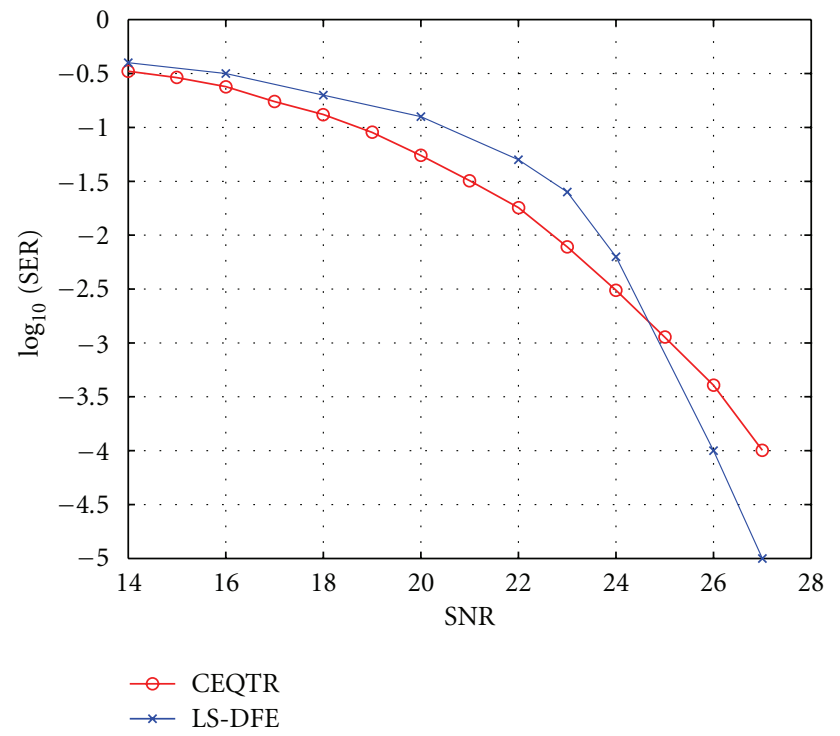

Figure 9: CEQTR, and LS-DFE Comparison, $L=768$, MaxNTap = $176, \alpha_{\max }=16$, FIR init $@ B_{L / 2}=1.0, \eta_{\mathrm{CMA}}=10^{-4}$ and $\eta_{\mathrm{DD}}=$ $10 \eta_{\mathrm{CMA}}$.

propagation in the DFE when operating under high noise levels.

\section{Conclusion}

This paper has proposed a novel adaptive concurrent equalizer with dynamic tap allocation as a low complexity solution for the blind channel deconvolution problem. Results have shown that the proposed equalizer is able to solve the blind channel deconvolution problem with a specified and reduced number of active taps in the equalizer filter, even when operating under an intense dynamic multipath scenario ( $f_{\text {doppler }}=150 \mathrm{~Hz}$ ). Not only does it minimize the cumulative noise which stems from a large number of inactive taps during and after the equalizer convergence, but also reduces the hardware implementation complexity.

\section{References}

[1] D. N. Godard, "Self-recovering equalization and carrier tracking in two dimensional data communication systems," IEEE transactions on communications systems, vol. 28, no. 11, pp. 1867-1875, 1980.

[2] F. C.C. De Castro, M. C.F. De Castro, and D. S. Arantes, "Concurrent blind deconvolution for channel equalization," in Proceedings of the IEEE International Conference on Communications (ICC'01), vol. 2, pp. 366-371, 2001.

[3] S. Chen and E. S. Chng, "Fractionally spaced blind equalization with low-complexity concurrent constant modulus algorithm and soft decision-directed scheme," International Journal of Adaptive Control and Signal Processing, vol. 19, no. 6, pp. 471-484, 2005.

[4] L. Ortolan, T. L. S. Santos, M. C. F. De Castro, and F. C. C. De Castro, "Blind concurrent beamformer," Electronics Letters, vol. 43 , no. 1 , pp. 5-7, 2007. 
[5] F. D’Agostini, S. Carboni Jr., M. C. F. De Castro, F. C. C. De Castro, and D. V. B. M. Trindade, "Adaptive concurrent equalization applied to multicarrier OFDM systems," IEEE Transactions on Broadcasting, vol. 54, no. 3, pp. 441-447, 2008.

[6] J. G. Proakis, Digital Communication, McGraw-Hill, New York, NY, USA, 2nd edition, 2001.

[7] J. L. Rojo-Àlvarez, M. Martínez-Ramón, J. Muñoz-Marí, G. Camps-Valls, C. M. Cruz, and A. R. Figueiras-Vidal, "Sparse deconvolution using support vector machines," EURASIP Journal on Advances in Signal Processing, vol. 2008, Article ID 816507, 2008.

[8] S. Gezici, Z. Sahinoglu, A. F. Molisch, H. Kobayashi, and H. V. Poor, "Two-step time of arrival estimation for pulse-based ultra-wideband systems," Eurasip Journal on Advances in Signal Processing, vol. 2008, Article ID 529134, 2008.

[9] I. Barhumi and M. Moonen, "Two-step time of arrival estimation for pulse-based ultra-wideband systems," EURASIP Journal on Advances in Signal Processing, vol. 2010, Article ID 704350, 2010.

[10] S. Baheci and M. Koca, "Iterative frequency-domain channel estimation and equalization for ultra-wideband systems with short cyclic prefix," EURASIP Journal on Advances in Signal Processing, vol. 2010, Article ID 819591, 2010.

[11] E. Önen, A. Akan, and L. F. Chaparro, "Time-frequency based channel estimation for high-mobility OFDM systemspart I: MIMO case," EURASIP Journal on Advances in Signal Processing, vol. 2010, Article ID 549197, 2010.

[12] X. Wei, D. G. M. Cruickshank, B. Mulgrew, and F. Riera-Palou, "A unified approach to dynamic length algorithms for adaptive linear equalizers," IEEE Transactions on Signal Processing, vol. 55, no. 3, pp. 908-920, 2007.

[13] L. Fan, C. He, D. Wang, and L. Jiang, "Efficient robust adaptive decision feedback equalizer for large delay sparse channel," IEEE Transactions on Consumer Electronics, vol. 51, no. 2, pp. 449-456, 2005.

[14] "Itu radiocommunication study groups: Document 6e/temp/ 131-e," Guidelines and Techniques for the Evaluation of DTTB Systems, March 2003.

[15] "Atsc digital television standard," ATSC Standard A/53, May 2004. 